\title{
Caracterización genotípica del virus de papiloma humano versus estudio citocolposcópico, hospital nacional dos de mayo, Perú. 2016-2018
}

\author{
Sandybel Quillama Palomino, ${ }^{1}$ (D) Carolina Cucho Espinoza, ${ }^{2}$ (D) Gloria Cruz Gonzales, ${ }^{3}$ \\ Regina Medina Espinoza, ${ }^{4}$ (ID) William Cruz Gonzales, ${ }^{5}$ (D) Aristides Hurtado Concha. ${ }^{6}$
}

\begin{abstract}
RESUMEN
Objetivo: Establecer la relación entre los genotipos del virus papiloma humano de alto riesgo con el grado de las lesiones citológicas y los hallazgos colposcópicas en mujeres del Hospital Nacional Dos de Mayo durante los años 2016-2018.

Métodos: Se realizó un estudio retrospectivo, tipo analítico de diseño no experimental. Para ello se revisaron 96 historias clínicas de pacientes con diagnóstico molecular positivo para virus papiloma humano de alto riesgo que cuenten con exámenes citológicos y colposcópicos, de ellos solo 76 casos cumplieron los criterios de inclusión.

Resultados: Las mujeres presentaron edades comprendidas entre 18 y 63 años, 33,38 $\pm 10,56$ años La frecuencia de virus papiloma humano de alto riesgo fue de $27,5 \%$, siendo el grupo de otros virus papiloma humano de alto riesgo los de mayor distribución con un 80,3\%. La lesión citológica más frecuente fue la lesión intraepitelial de bajo grado con un 61,85\%. Los hallazgos colposcópicos más frecuentes fueron los menores con el 76,3\%.

Conclusión: Se encontró una alta frecuencia del virus papiloma humano, tipos 16, 18 y otros virus papiloma humano de alto riesgo, en ese orden de frecuencia. Se identificó que la lesiones intraepiteliales cervicales más frecuente fueron las de bajo grado, los hallazgos de evaluación colposcópica más frecuentes en mujeres con virus de alto riesgo positivo fueron menores. El pico de infección por virus papiloma humano de alto riesgo estuvo en el grupo de 18 a 29 años.
\end{abstract}

Palabras clave: Virus del Papiloma Humano, Colposcopia, Citologías.

\section{SUMMARY}

Objective: Establish the relationship between the genotypes of the high-risk Human Papillomavirus with the degree of cytological lesions and colposcopic findings in women from the Hospital Nacional Dos de Mayo during the years 2016-2018.

Methods: A retrospective study was carried out, analytical type of non-experimental design. For this, 96 clinical records of patients with a positive molecular diagnosis for high-risk Human Papillomavirus who have cytological and colposcopic examinations were reviewed, of which only 76 cases met the inclusion criteria.

Results: The women presented ages between 18 and 63 years, $33.38 \pm 10.56$ years. The frequency of high-risk Human Papillomavirus was $27.5 \%$, being the group of other high-risk Human Papillomavirus the ones with the highest distribution with $80.3 \%$. The most frequent cytological lesion was low grade squamous intraepithelial lesions with $61.85 \%$. The most frequent colposcopic findings are the minor ones with $76.3 \%$.

Conclusion: A high frequency of human papillomavirus, types 16, 18 and other high-risk human papillomaviruses, was found in that order of frequency. It was identified that the most frequent cervical intraepithelial lesions were those of low grade, the most frequent colposcopic evaluation findings in women with positive high-risk viruses were lower. The peak of high-risk human papillomavirus infection was in the 18-29 age group.

Key words: Human Papillomavirus, Colposcopy, Cytologies.

\section{INTRODUCCIÓN}

${ }^{1}$ Licenciado Tecnólogo médico en Laboratorio y Anatomía patológica. Universidad Nacional Federico Villarreal. ${ }^{2}$ Maestra en Ciencias básicas con mención en Bioquímica Universidad Nacional Mayor de San Marcos. ${ }^{3}$ Doctora en Salud Pública. Universidad Nacional Federico Villarreal. ${ }^{4}$ Doctora en Salud Pública. Universidad Nacional Federico Villarreal. ${ }^{5}$ Doctor en Contabilidad. Universidad Nacional Federico Villarreal. 'Doctor en Psicología educacional y tutorial. Universidad Nacional de Educación Enrique Guzmán y Valle.

*El trabajo no ha sido presentado en alguna reunión o congreso, sirvió para obtener Titulo profesional. No recibió ayuda financiera.
El virus del papiloma humano (VPH) produce infecciones de transmisión sexual en genitales, ano $\mathrm{y}$ orofaringe, zonas en las que puede provocar la aparición de tumores malignos. Se trata de un virus común, generalmente causa una infección silente que, después de años de evolución puede transformar las 


\section{CARACTERIZACIÓN GENOTÍPICA DEL VIRUS DE PAPILOMA HUMANO VERSUS ESTUDIO CITOCOLPOSCÓPICO, HOSPITAL NACIONAL DOS DE MAYO, PERÚ. 2016-2018}

células epiteliales en malignas (1). El VPH forma parte de un grupo de virus ácido desoxirribonucleico (ADN) heterogéneo llamados papillomaviridae; este virus, es causante de múltiples lesiones hiperplásicas, verrugosas y papilomatosas de las células epiteliales de piel y mucosas (1). La mayoría de los hombres y mujeres contraen la infección poco después del inicio de la vida sexual. El VPH se transmite por vía sexual, si bien no es necesario que haya una relación sexual con penetración para que se produzca la transmisión, también el contacto directo piel con piel de la zona genital es un modo de transmisión reconocido (2).

El VPH, ocupa el cuarto lugar entre los tipos más frecuentes de cáncer que afectan a mujeres, con un número estimado de 266 mil muertes y unos $528 \mathrm{mil}$ nuevos casos. El mayor porcentaje (alrededor del $85 \%$ ) de esas muertes se produjeron en las regiones menos desarrolladas, donde representa aproximadamente el $12 \%$ de todos los cánceres femeninos. Prácticamente todos los casos de cáncer de cuello de útero (el 99\%) están vinculados con la infección genital por el VPH, que es la infección vírica más común del aparato reproductor. El VPH también puede causar otros tipos de cáncer anogenital, cánceres de cabeza y cuello y verrugas genitales tanto en hombres como en mujeres (2).

En el Perú, el Instituto Nacional de Enfermedades Neoplásicas (INEN) resalta que la neoplasia cervical constituye el cáncer más frecuente, cuya causa más común es la infección por el VPH, con tasas de incidencia que han aumentado entre los periodos 2000-2017. En este sentido, en el Perú se han tomado medidas preventivas primarias y secundarias con el fin de disminuir considerablemente el número de casos de muertes en mujeres por este tipo de cáncer (3).

Aunque la mayor parte de las infecciones por VPH no provocan síntomas, la infección genital por VPH persistente puede causar cáncer de cuello de útero en las mujeres. Las pruebas rutinarias que se han usado para el diagnóstico del carcinoma son la citología y la biopsia. Estas han demostrado reducir la incidencia del mismo. Dentro de las pruebas diagnósticas de rutina para la infección por el VPH y la lesión intraepitelial escamosa (LIE), están la biopsia, que muestra los cambios histológicos en el tejido, y las pruebas moleculares que, debido a que el VPH no puede ser cultivado in vitro, se fundamentan en la secuencia de ADN; por ejemplo, hibridación in situ, Southern blot, captura de híbridos y reacción en cadena de la polimerasa (PCR) (4).

Inicialmente, los métodos de diagnóstico que se utilizaron fueron enteramente de tipo morfológico, como la citología y colposcopia, sin embargo, tras 50 años de la utilización de la citología como método de tamizaje para cáncer cervical, junto a la colposcopia, como método de verificación, no han logrado reducir la mortalidad. La citología es un método de diagnóstico sencillo, económico y aplicable a grandes sectores de la población. No obstante, la baja sensibilidad del método puede diferir en forma importante de acuerdo con diversas variables que pueden incluir desde la adecuada toma de muestra, la tinción y la lectura, hasta el control de calidad. Posteriormente surgieron otros métodos como la detección directa del virus por métodos moleculares más sensibles como la PCR (reacción en cadena de la polimerasa), estas nuevas metodologías no solo permitieron identificar, sino también tipificar, genotipos de VPH para así tener una mejor orientación terapéutica y preventiva (5).

El presente trabajo de investigación tiene como objetivo fundamental establecer la relación entre los genotipos del virus del papiloma humano de alto riesgo con el grado de las lesiones citológicas y los hallazgos colposcópicos en mujeres del Hospital Nacional Dos de Mayo, durante los años 2016-2018. 


\section{MÉTODOS}

La investigación fue de tipo analítica, de diseño retrospectivo, no experimental. Se procesaron los datos obtenidos de los siguientes estudios: citología, colposcopía y molecular de mujeres atendidas durante el periodo agosto de 2016 hasta agosto de 2018, en los consultorios externos del servicio de Ginecología del Hospital Nacional Dos de Mayo (HNDM), Lima, Perú.

Se consideraron los siguientes criterios de inclusión: mujeres con infección de VPH-AR, con estudio citológico para cáncer de cuello uterino (CCU) y estudio colposcópico para CCU. No se consideraron a las pacientes con resultados de citología, colposcopia o estudio molecular que estuvieran incompletos o poco legibles en la historia clínica y, por lo tanto, fueron excluidas del estudio.

Se realizó un muestreo consecutivo en el área de biología molecular del HNDM que recibió un total de 349 solicitudes para la determinación de VPH, de las cuales se obtuvo 96 VPH-positivas y $253 \mathrm{VPH}$ negativas. Para este estudio solo se consideraron los resultados positivos para VPH-AR, de los cuales solo 76 cumplían con los criterios de inclusión.

Se elaboró una ficha de recolección de datos con base en las variables genotipos del VPH-AR, estudio citológico, estudio colposcópico. Se revisaron las historias clínicas y la base de datos del departamento de patología clínica y anatomía patológica (servicio de biología molecular), seleccionadas de acuerdo con el periodo y a los criterios de inclusión y exclusión ya establecidos.

El Sistema Cobas ${ }^{\circledR} 4800$ Human Papilomavirus (HPV) es una prueba in vitro de tipo cualitativo que permite identificar la presencia de 14 tipos de HPV de alto riesgo: $16,18,31,33,35,39,45,51,52,56,58$,
59, 66 y 68. Se trata de una técnica de amplificación de secuencias de DNA diana mediante una reacción en cadena de la polimerasa (PCR) y una hibridación de ácidos nucleicos. La prueba permite identificar específicamente los tipos 16 y 18 de modo individual (positivo o negativo para cada uno de los dos), y el resto de los tipos de forma agrupada (positivo o negativo para cualquiera de ellos).

La observación e interpretación de la colposcopia la realiza el ginecólogo. La toma de muestra y la fijación de la citología la realiza el ginecólogo, mientras que la coloración y la lectura es responsabilidad del personal de laboratorio y anatomía patológica.

Se utilizaron los programas Microsoft Office Excel 2016 y SPSS para Windows versión 24. Se utilizó el estadístico exacto de Fisher en los cruces: VPH 16 $\mathrm{x}$ edad, VPH $18 \mathrm{x}$ edad, VPH otros x edad. Se usó la prueba de Chi-cuadrado en los cruces: citología $\mathrm{x}$ edad, colposcopia x edad.

El protocolo de investigación fue aprobado por la Oficina de Grados y Títulos de la Facultad de Tecnología Médica de la Universidad Nacional Federico Villarreal del Perú, además existió la autorización del Comité de Ética del Hospital Nacional Dos de Mayo.

\section{RESULTADOS}

Se incluyeron 76 resultados de determinación de VPH. Las pacientes presentaron edades comprendidas entre 18 y 63 años, la media aritmética y desviación estándar fue de 33,38 $\pm 10,56$ años, error estándar de 1,2 años.

En la tabla 1 se observa la distribución de los genotipos de VPH. El VPH 16 se encontró en 30,3 \% ( $n=23)$, el VPH 18 en 3,9 \% (n=3) y otros tipos de VPH en $80,3 \%(\mathrm{n}=61)$. También se encontró que 8 pacientes $(10,5 \%)$ presentaban coinfección entre VPH 16 y 


\section{CARACTERIZACIÓN GENOTÍPICA DEL VIRUS DE PAPILOMA HUMANO VERSUS ESTUDIO CITOCOLPOSCÓPICO, HOSPITAL NACIONAL DOS DE MAYO, PERÚ. 2016-2018}

Tabla 1. Relación entre los genotipos del virus de papiloma humano de alto riesgo y el estudio citológico

\begin{tabular}{|c|c|c|c|c|}
\hline \multirow[b]{2}{*}{ Genotipo viral } & \multicolumn{3}{|c|}{ Citología } & \multirow[b]{2}{*}{$\mathrm{p}$} \\
\hline & $\begin{array}{c}\text { Negativo } \\
n(\%) \\
n=26\end{array}$ & $\begin{array}{c}\text { Lesión intraepitelial } \\
\text { de bajo grado } \\
\mathrm{n}(\%) \\
\mathrm{n}=47\end{array}$ & $\begin{array}{c}\text { Lesión intraepitelial } \\
\text { de alto grado } \\
\mathrm{n}(\%) \\
\mathrm{n}=3\end{array}$ & \\
\hline 16 & & & & 0,068 \\
\hline Positivo $n=23$ & $4(5,3)$ & $17(22,4)$ & $2(2,6)$ & \\
\hline Negativo $n=53$ & $22(28,9)$ & $30(39,5)$ & $1(1,3)$ & \\
\hline 18 & & & & 0,934 \\
\hline Positivo $n=3$ & $1(1,3)$ & $2(2,6)$ & 0 & \\
\hline Negativo $n=73$ & $25(32,9)$ & $45(59,2)$ & $3(3,9)$ & \\
\hline Otros & & & & 0,833 \\
\hline Positivo $n=61$ & $21(27,6$ & $38(50)$ & $2(2,6)$ & \\
\hline Negativo $n=15$ & $5(6,6)$ & $9(11,8)$ & $1(1,3)$ & \\
\hline
\end{tabular}

otros VPH-AR, mientras que solo una paciente $(1,3 \%)$ tenía la presencia simultánea de VPH 16, 18 y otros de alto riesgo. También se puede observar en la misma tabla que la mayoría de los estudios citológicos, fueron positivos para lesiones intraepiteliales escamosas de bajo grado (LIEBG), con el 61,8 \% ( $\mathrm{n}=47)$, se agrupó el diagnóstico citológico de negativo para lesión intraepitelial, células escamosas atípicas de significado indeterminado (ASCUS) e inflamación y representó el 34,2\% $(n=26)$, en tercer lugar se presentó el diagnóstico citológico del tipo lesión intraepitelial escamosa de alto grado (LIEAG) con el 3,9\% $(n=3)$. No hubo relación estadísticamente significativa entre el genotipo del VPH-AR y el estudio citológico ( $p>$ $0,05)$.

En la tabla 2 se cruzaron genotipos VPH-AR y estudio colposcópico. Se observó que del total $(n=23)$ de VPH 16, la mayor frecuencia fue de cambios colposcópicos menores $(n=17)$. Con respecto al VPH 18 , todos los casos presentaron cambios menores en el estudio colposcópico y, finalmente, en los otros tipos de VPH $(\mathrm{n}=61)$ se encontró nuevamente que predominaron los cambios menores $(n=46)$. No se encontró asociación estadísticamente significativas $(\mathrm{p}>0,05)$ entre genotipo de VPH y cambio colposcópico.

En la tabla 3 se cruzaron genotipos de VPH-AR, el estudio citológico y el estudio colposcópico con la edad. No se encontraron diferencias estadísticamente significativas a un nivel de confianza del $95 \%$ (p > 0,05). La mayor frecuencia de VPH 16 estuvo en las mujeres menores o iguales a 30 años $(n=14)$, mientras que el VPH $18(n=2)$ y otros tipos de VPH $(n=35)$ se encontraron con más frecuencia en las pacientes de 31 años o más. Con relación a la citología se obtuvo que las usuarias con LIEBG $(n=27)$ y LIEAG $(n=2)$ se observaron con más frecuencia en mujeres de 31 años o más. Con respecto a la colposcopia la mayor frecuencia en los cambios menores se vio en mujeres mayores o iguales a 31 años $(n=30)$, mientras que la mayor frecuencia en los cambios mayores se vio en las mujeres menores o iguales a 30 años $(n=4)$. 
S QUILLAMA PALOMINO ET AL.

Tabla 2. Relación entre el genotipo de virus de papiloma humano de alto riesgo y el estudio colposcópico

\begin{tabular}{cccccc}
\hline & \multicolumn{5}{c}{ Colposcopia } \\
\cline { 2 - 5 } Genotipo viral & $\begin{array}{c}\text { No adecuada } \\
\mathrm{n}(\%) \\
\mathrm{n}=3\end{array}$ & $\begin{array}{c}\text { Normales } \\
\mathrm{n}(\%) \\
\mathrm{n}=8\end{array}$ & $\begin{array}{c}\text { Cambios } \\
\text { menores } \mathrm{n}(\%) \\
\mathrm{n}=58\end{array}$ & $\begin{array}{c}\text { Cambios } \\
\text { mayores } \mathrm{n}(\%) \\
\mathrm{n}=7\end{array}$ & $\mathrm{p}$ \\
\hline 16 & 0 & $3(3,9)$ & 17 & $3(3,9)$ & 0,562 \\
Positivo $\mathrm{n}=23$ & $3(3,9)$ & $5(6,6)$ & 41 & $4(5,3)$ & \\
Negativo $\mathrm{n}=53$ & & & & & 0,809 \\
18 & 0 & 0 & 3 & 0 & \\
Positivo $\mathrm{n}=3$ & $3(3,9)$ & $8(10,5)$ & 55 & $7(9,2)$ & \\
Negativo $\mathrm{n}=73$ & & & & & \\
Otros & $3(3,9)$ & $7(9,2)$ & 46 & $5(6,6)$ & \\
Positivo $\mathrm{n}=61$ & 0 & $1(1,3)$ & 12 & $2(2,6)$ & \\
Negativo $\mathrm{n}=15$ & & & & & \\
\hline
\end{tabular}

Tabla 3. Relación entre el genotipo de virus de papiloma humano de alto riesgo y el estudio colposcópico

\begin{tabular}{|c|c|c|c|}
\hline \multirow[t]{2}{*}{ Genotipo viral } & \multicolumn{2}{|c|}{ Grupo de edad (años) } & \multirow[t]{2}{*}{$\mathrm{p}$} \\
\hline & $\begin{array}{c}\leq 30 \text { años } \\
\mathrm{n}(\%) \mathrm{n}=36\end{array}$ & $\begin{array}{l}\geq 31 \text { años } \\
\mathrm{n}(\%) \mathrm{n}=40\end{array}$ & \\
\hline 16 & & & 0,140 \\
\hline Positivo $n=23$ & $14(18,4)$ & $9(11,8)$ & \\
\hline Negativo $n=53$ & $22(28,9)$ & $31(40,8)$ & \\
\hline 18 & & & 1,000 \\
\hline Positivo $n=3$ & $1(1,3)$ & $2(2,6)$ & \\
\hline Negativo $n=73$ & $35(46,1)$ & $38(50)$ & \\
\hline Otros & & & 0,148 \\
\hline Positivo $n=61$ & $26(34,2)$ & $35(46,1)$ & \\
\hline Negativo $n=15$ & $10(13,2)$ & $5(6,6)$ & \\
\hline Citología & & & 0,409 \\
\hline Negativa $n=26$ & $15(19,7)$ & $11(14,5)$ & \\
\hline LIE bajo grado $n=47$ & $20(26,3)$ & $27(35,5)$ & \\
\hline LIE alto grado $n=3$ & $1(1,3)$ & $2(2,6)$ & \\
\hline Colposcopia & & & 0,390 \\
\hline No adecuada $n=3$ & 0 & $3(3,9)$ & \\
\hline Normales $n=8$ & $4(5,3)$ & $4(5,3)$ & \\
\hline Cambios menores $\mathrm{n}=58$ & $28(36,8)$ & $30(39,5)$ & \\
\hline Cambios mayores $n=7$ & $4(5,3)$ & $7(9,2)$ & \\
\hline
\end{tabular}

\section{DISCUSIÓN}

En el presente estudio se obtuvo una frecuencia de infección por virus de papiloma humano del 27,5\%, en un total de 349 pacientes, mediante PCR en tiempo real. Estos resultados son semejantes a los obtenidos por Iwasaki y cols. (6) quienes analizaron 2247 muestras cervicales, mediante el Cobas $4800 \mathrm{VPH}$ Test y obtuvo una frecuencia del 34,49\% de VPHAR positivo. Estas altas frecuencias podrían estar explicando el aumento de los casos de mujeres con cáncer de cuello uterino, ya que se ha determinado que existe una relación directa entre la prevalencia de la infección por el VPH y la incidencia de cáncer cervical (7).

Bobadilla y cols. (8) mencionaron que el VPH 16 es el más frecuente, esto coincide con este estudio en el cual se encontró un 30,3\% entre los casos con VPH-AR, mientras que el VPH 18 representó solo el 3,9\% de los casos. Con respecto a los otros genotipos de VPH$\mathrm{AR}$, se encontró en primer lugar con un $80,3 \%$ de los casos considerados en este estudio. Estos resultados son semejantes a los obtenidos por Iwasaki y cols. (6) 


\section{CARACTERIZACIÓN GENOTÍPICA DEL VIRUS DE PAPILOMA HUMANO VERSUS ESTUDIO CITOCOLPOSCÓPICO, HOSPITAL NACIONAL DOS DE MAYO, PERÚ. 2016-2018}

quienes obtuvieron una alta frecuencia de los otros VPH-AR. Las variantes moleculares especialmente de los genotipos 16 y 18 , son determinantes de importancia de progresión a lesiones de alto grado del cuello uterino (9). En este estudio, se encontró que las pacientes con genotipos 16 y 18 presentaban, en su mayoría, citología y colposcopia positiva, a diferencia de las que tenían otros VPH-AR que presentaban, en su mayoría, diagnóstico citológico y colposcópico negativo. Este trabajo demostró asociación entre algunos resultados citológicos y la positividad al VPH-AR. Sin embargo, cuando la citología indicaba un resultado negativo la asociación entre los métodos disminuyó, esto debido a la baja sensibilidad de la citología sobre las pruebas moleculares.

En cuanto a la colposcopia con resultados negativos, coincide con la evolución de la enfermedad porque en los primeros estadios, se produce una infección sin cambios hasta que el virus se integra al ADN celular, evento en el que se producen los cambios visualizados por la colposcopia (10). Esto refleja una baja sensibilidad con relación a la detección del VPH por PCR.

En el presente estudio se evidenció que las pacientes presentaron edades comprendidas entre 18 y 63 años con una media de 33,38 $\pm 10,56$ años. El inicio de las relaciones sexuales en la mujer joven hace que se adquiera rápidamente el virus, luego el virus pasa a estado de latencia por eso la incidencia baja y posteriormente el aumento de incidencia en mujeres mayores quizás se deba a la reactivación del virus. Estudios de cohortes en Estados Unidos e Inglaterra, en los que se hizo el seguimiento de adolescentes de 15 a 19 años al comenzar su actividad sexual y con muestras de células cervicales para la detección de ADN de VPH cada 4-6 meses, demostraron que a los 4 años $50 \%$ a $60 \%$ de los individuos había adquirido la infección y que el intervalo promedio entre el primer coito y el descubrimiento de VPH es de alrededor de 3 meses (11).

Vol. 81, $\mathrm{N}^{\mathrm{o}} 2$, junio 2021
La investigación muestra en las pacientes estudiadas una alta frecuencia de VPH, además se observó la presencia de VPH 16, VPH 18 y otros VPH-AR en ese orden de frecuencia, también se identificó que la lesión cervical más frecuente fue la LIEBG, los hallazgos de evaluación colposcópica más frecuentes en mujeres con VPH-AR positivo son los hallazgos colposcópicos menores. Finalmente se obtuvo que la presencia del VPH-AR se asocia con la presencia de ciertas alteraciones citológicas y hallazgos colposcópicos. Sin embargo, con respecto al grado de lesión citológica y colposcópica que puede generar un determinado genotipo de VPH-AR, el estudio no da resultados concluyentes ya que el resultado final de una infección por VPH no solo está determinado por el tipo de VPH, sino también por la respuesta inmune del huésped y por otros cofactores.

Se recomienda realizar estudios considerando más casos y también a los otros cofactores asociados al cáncer de cuello uterino como la multiparidad, el uso prolongado de anticonceptivos orales, el consumo de tabaco y la infección previa de otros agentes infecciosos.

Que los establecimientos de salud incluyan un diagnóstico molecular que permita identificar los genotipos de alto riesgo de VPH como prueba de tamización ya que ofrecería la posibilidad de mejorar la eficiencia en el manejo de cáncer de cuello uterino.

Que las mujeres vacunadas se hagan una prueba molecular para prevenir el cáncer causado por los otros genotipos no incluidos en las vacunas y a su vez se debería tipificar los otros VPH-AR para conocer los tipos virales circulantes en la población.

Promocionar el diagnóstico temprano de cáncer de cuello uterino, especialmente en el grupo de mujeres jóvenes. 


\section{REFERENCIAS}

1. Cháirez P, Vega M, Zambrano G, Garcia A, Maya I, Cuevas J. Presencia del virus papiloma humano en la cavidad oral: revisión y actualización de la literatura. Int J Odontostomat [Internet]. 2015 [consultado 06 agosto 2019 ]; 9(2):233-238. Disponible en: https:// scielo.conicyt.cl/pdf/ijodontos/v9n2/art09.pdf.

2. Organización Mundial de la Salud [Internet]. Ginebra: Papilomavirus Humanos (HPV) y Cáncer Cervicouterino; 2019. [consultado 10 septiembre 2019] Disponible en: https://www.who.int/es/news-room/ fact-sheets/detail/human-papillomavirus-(hpv)-andcervical-cancer.

3. Instituto Nacional de Enfermedades Neoplásicas [Internet]. Indicadores anuales de gestión producción hospitalaria: 2018. [consultado 15 agosto 2019] Disponible en: https://portal.inen.sld.pe/indicadoresanuales-de-gestion-produccion-hospitalaria.

4. Colín M, Domínguez M, Mendieta H, Rojas I, Romero M. Sensibilidad y especificidad de las pruebas de citología, colposcopia, biopsia y detección del virus del papiloma humano en lesión intraepitelial escamosa [Internet]. México: Universidad Autónoma del Estado de México y Ediciones y Gráficos Eón; 2017 [consultado 10 agosto 2019]. Disponible en: http:// ri.uaemex.mx/handle/20.500.11799/67963

5. Li W, Padilla C, Gutierrez E, Hijar G. Detection molecular y genotipificación del virus del papilloma humano como tamizaje de cáncer de cuello uterino: posibilidades en el contexto peruano. Bol Inst Nac Salud [Internet]. 2016 [consultado 10 septiembre 2019 ]; 22(1-3):22-28. Disponible: https://repositorio.ins. gob.pe/xmlui/bitstream/handle/INS/907/2016\%2813\%2922-28.pdf?sequence $=1 \&$ is Allowed $=\mathrm{y}$.

6. Iwasaki R, Arias-Stela J Jr, Arias-Stela J. Prevalencia del virus del papiloma humano de alto riesgo en el Perú. Diagnóstico [Internet]. 2014 [consultado 06 de agosto 2019]; 53(1):5-8. Disponible en: http://repebis. upch.edu.pe/articulos/diag/v53n1/a2.pdf
7. Clifford G., Gallus S., Herrero R., Muñoz N., Snijders P., Vaccarella S, et al. Worldwide distribution of human papillomavirus types in cytologically normal women in the International Agency for Research on Cancer HPV prevalence surveys: a pooled analysis. Lancet. 2005; 366:991-998. doi: 10.1016/S0140-6736(05)67069-9.

8. Bobadilla M, Villagra V, Zorrilla M, Olmedo G, Riveros M, Franco F, et al. Detección y tipificación del Virus Papiloma Humano en el marco del tamizaje virológico para la detección de lesiones de cuello uterino en Asunción, Paraguay. Mem Inst Investig Cienc Salud. Paraguay [Internet]. 2019 [consultado 20 de agosto del 2019]; 17(1):6-15. Disponible: http://scielo.iics.una. py/pdf/iics/v17n1/1812-9528-iics-17-01-6.pdf

9. Sichero L, Ferreira S, Trottier H, Duarte-Franco E, Ferenczy A, Franco EL, et al. High grade cervical lesions are caused preferentially by non-European variants of HPVs 16 and 18. Int J Cancer. 2007; 120(8):1763-1768. doi: 10.1002/ijc.22481.

10. Shah KV, Stern WF, Shah FK, Bishai D, Kashima HK. Risk factors for juvenile onset recurrent respiratory papillomatosis. Pediatr Infect Dis J. 1998; 17(5):372376. doi: 10.1097/00006454-199805000-00005.

11. Winer RL, Lee SK, Hughes JP, Adam DE, Kiviat NB, Koutsky LA. Genital human papillomavirus infection: incidence and risk factors in a cohort of female university students. Am J Epidemiol. 2003; 157(3):218-226. doi: 10.1093/aje/kwf180. Erratum in: Am J Epidemiol. 2003 May 1;157(9):858.

Recibido 24 de octubre de 2020 Aprobado 18 de enero de 2021 